\title{
Asymptotic approximation of long-time solution for low-temperature filtration combustion
}

\author{
Grigori Chapiro - Alexei A. Mailybaev - Aparecido J. de Souza • \\ Dan Marchesin • Johannes Bruining
}

Received: 22 April 2011 / Accepted: 1 March 2012 / Published online: 21 March 2012

(C) Springer Science+Business Media B.V. 2012

\begin{abstract}
There is a renewed interest in using combustion for the recovery of medium viscosity oil. We consider the combustion process when air is injected into the porous medium containing some fuel and inert gas. Commonly the reaction rate is negligible at low temperatures, hence the possibility of oxygen breakthrough. In this case, the oxygen gets in contact with the fuel in the downstream zone leading to slow reaction. We focus on the case when the reaction is active for all temperatures, but heat losses are negligible. For a combustion model that includes heat and mass balance equations, we develop a method for calculating the wave profile in the form of an asymptotic expansion
\end{abstract}

G. Chapiro $(\varangle)$

UFJF, Juiz de Fora, Brazil

e-mail: grigorichapiro@gmail.com

A. A. Mailybaev · D. Marchesin

IMPA, Rio de Janeiro, Brazil

A. A. Mailybaev

e-mail: alexei@impa.br

D. Marchesin

e-mail: marchesi@fluid.impa.br

A. A. Mailybaev

Moscow State University, Moscow, Russia
A. J. de Souza
UFCG, Campina Grande, Brazil
e-mail: cido@dme.ufcg.edu.br
J. Bruining
TU Delft, Delft, the Netherlands
e-mail: j.bruining@tudelft.nl

and derive its zero- and first-order approximations. This wave profile appears to be different from wave profiles analyzed in other papers, where only the reaction at the highest temperatures was taken into account. The combustion wave has a long decaying tail. This tail is hard to observe in the laboratory because heat losses must be very small for the long tail to form. Numerical simulations were performed in order to validate our asymptotic formulae.

Keywords Filtration combustion - Traveling wave • Singular perturbation - Low-temperature oxidation . Asymptotic expansions

Mathematics Subject Classifications (2010) 80A25 • 76S05

\section{Introduction}

In this paper, we consider the combustion process when air is injected into porous medium containing initially some fuel (e.g., oil) and inert gas. There are many analytical studies of steady co-flow combustion waves in high-temperature regimes, e.g., [2-5, 14, 15, 17]. The method in these papers usually exploits strong nonlinearity of the Arrhenius factor in the reaction rate, which allows neglecting the reaction rate as soon as the temperature decreases [19]. This method is valid provided that most of the reaction occurs at the highest temperatures. One of the consequences of this assumption is that oxygen is not consumed completely at the highest temperatures and its breakthrough becomes possible. In such a case, the oxygen gets in contact with fuel 
downstream of the fast reaction zone, leading to slow reaction in the downstream zone. In many practical applications, this reaction rate is extremely low, and the generated heat dissipates to the surrounding media. This is the main reason for neglecting reaction at low temperatures, e.g., in laboratory experiments. However, for field applications, there are low-temperature oxidation reactions, which are relatively fast, as well as heat losses are very small.

In this paper, we focus on the latter case, i.e., when the reaction is active for all temperatures, but heat losses are negligible. For a combustion model that includes heat and mass balance equations, we develop a method for computing the wave profile in the form of an asymptotic expansion. In particular, we derive formulae for zero- and first-order approximations of the profile. It turns out that the wave has a long decaying tail. Due to its long span, such a combustion wave may exist in field experiments, but not in laboratory conditions, because this tail occurs only if heat losses are very small. To justify our results, we perform direct numerical simulations of the original system. The results of the numerical simulations are in perfect agreement with our asymptotic formulae.

The paper is organized as follows: In Section 2, we describe the physical model for in situ combustion and its dimensionless form. In Section 3, we describe the solution of the problem as a wave sequence. In Section 4 , we derive the zero-order asymptotic expression for the combustion wave profile. In Section 5, numerical computations and simulations are carried out for the case of low-temperature oil oxidation. The results are summarized in the Section 6. In the Appendix, we present the details about asymptotic expansion for the wave profile and compute its zero and first-order terms using the singular perturbation method.

\section{The model}

We study one-dimensional flows possessing a combustion wave in the case when the oxidizer (air with oxygen) is injected in the porous medium, see Fig. 1. The medium contains initially a fuel that is essentially immobile and does not vaporize and a non-reactive gas. This is the case for solid fuel, or for liquid fuel at low saturations so it does not move. In our simplified model, we assume that only a small part of the available space is occupied by the fuel, so that changes of porosity in the reaction are negligible. We assume

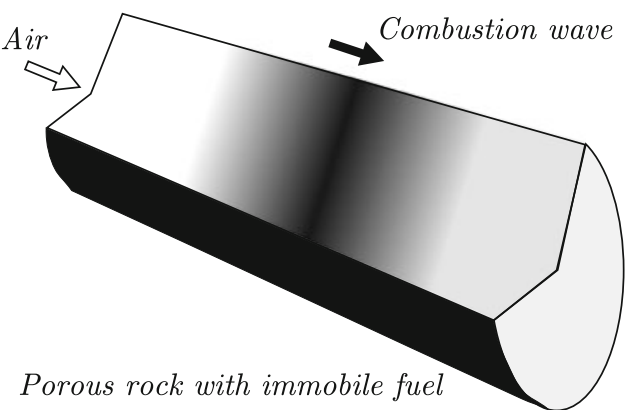

Fig. 1 Sketch of in situ combustion

that the temperature of solid and gas is the same (local thermal equilibrium). Heat losses are neglected, which is reasonable for in situ combustion in field conditions. We also assume that pressure variations are small compared to prevailing pressure.

The model with time coordinate $t$ and space coordinate $x$ includes the heat balance Eq. 1, the molar balance equations for total gas (Eq. 2), oxygen (Eq. 3), immobile fuel (Eq. 4), and the ideal gas law (Eq. 5):

$C_{\mathrm{m}} \frac{\partial T}{\partial t}+\frac{\partial\left(c_{\mathrm{g}} \rho u\left(T-T_{\text {res }}\right)\right)}{\partial x}=\lambda \frac{\partial^{2} T}{\partial x^{2}}+Q_{\mathrm{r}} W_{\mathrm{r}}$

$\varphi \frac{\partial \rho}{\partial t}+\frac{\partial(\rho u)}{\partial x}=\left(\mu_{\mathrm{g}}-\mu_{\mathrm{o}}\right) W_{\mathrm{r}}$

$\varphi \frac{\partial(Y \rho)}{\partial t}+\frac{\partial(Y \rho u)}{\partial x}=D \varphi \frac{\partial}{\partial x}\left(\rho \frac{\partial Y}{\partial x}\right)-\mu_{\mathrm{o}} W_{\mathrm{r}}$,

$\frac{\partial \rho_{\mathrm{f}}}{\partial t}=-\mu_{f} W_{r}$,

$T=P /(\rho R)$.

Here $T$ (kelvin) is the temperature, $\rho$ (moles per cubic meter) is the molar density of gas, $Y$ (moles per mole) is the oxygen molar fraction in the gas, $u$ (meters per second) is the Darcy velocity of gas, $\rho_{\mathrm{f}}$ (moles per cubic meter) is the molar concentration of immobile fuel. The system parameters together with their typical values are given in Table 1. These parameters are assumed to be constant (neglecting the dependence on temperature, gas composition, etc.), an assumption that was already used in Eq. 1. 
Table 1 Dimensional parameters for in-situ combustion and their typical values

\begin{tabular}{llll}
\hline Symbol & Physical quantity & Value & $\mathrm{Unit}$ \\
\hline$T_{\text {res }}$ & Initial reservoir temperature & 370 & $\mathrm{~K}$ \\
$C_{\mathrm{m}}$ & Heat capacity of porous medium & $2 \times 10^{6}$ & $\mathrm{~J} / \mathrm{m}^{3} \mathrm{~K}$ \\
$c_{\mathrm{g}}$ & Heat capacity of gas & 27.42 & $\mathrm{~J} / \mathrm{mol} \mathrm{K}^{6}$ \\
$\lambda$ & Thermal conductivity of porous medium & 0.87 & $\mathrm{~J} /(\mathrm{m} \mathrm{s} \mathrm{K})$ \\
$Q_{\mathrm{r}}$ & Immobile fuel combustion enthalpy at $T_{\mathrm{res}}$ & $4 \times 10^{5}$ & $\mathrm{~J} / \mathrm{mol}$ \\
$\varphi$ & Porosity & 0.3 & $\cdot$ \\
$D$ & Gas diffusion coefficient & $2 \times 10^{-6}$ & $\mathrm{~m}^{2} / \mathrm{s}$ \\
$E_{\mathrm{r}}$ & Activation energy & 58,000 & $\mathrm{~J} / \mathrm{mol}$ \\
$k_{\mathrm{p}}$ & Pre-exponential parameter & 500 & $1 / \mathrm{s}$ \\
$R$ & Ideal gas constant & 8.314 & $\mathrm{~J} /(\mathrm{mol} \mathrm{K})$ \\
$P$ & Prevailing pressure (1 atm) & 101,325 & $\mathrm{~Pa}$ \\
$u_{\text {inj }}$ & Darcy velocity of injected gas $(200 \mathrm{~m} /$ day $)$ & $2.3 \times 10^{-3}$ & $\mathrm{~m} / \mathrm{s}$ \\
$Y_{\text {inj }}$ & Oxygen molar fraction in injected gas & 0.21 & $\cdot$ \\
$\rho_{\mathrm{f}}^{\text {res }}$ & Initial molar density of fuel & 372 & $\mathrm{~mol} / \mathrm{m}^{3}$ \\
\hline
\end{tabular}

In the combustion reaction, $\mu_{\mathrm{f}}$ moles of immobile fuel react with $\mu_{\mathrm{o}}$ moles of oxygen and generate $\mu_{\mathrm{g}}$ moles of gaseous products and, possibly, unreactive solid products. For simplicity, we consider the case $\mu_{\mathrm{f}}=$ $\mu_{\mathrm{o}}=\mu_{\mathrm{g}}=1$ as, e.g., in the reaction $\mathrm{C}+\mathrm{O}_{2} \rightarrow \mathrm{CO}_{2}$. The reaction rate $W_{\mathrm{r}}$ will be taken as

$W_{\mathrm{r}}=k_{\mathrm{p}} Y \rho_{\mathrm{f}} \exp \left(-\frac{E_{\mathrm{r}}}{R T}\right)$,

where typical values of $k_{\mathrm{p}}$ and $E_{\mathrm{r}}$ also are given in Table 1.

The variables to be found are the temperature $T$, the molar concentration of immobile fuel $\rho_{\mathrm{f}}$, the molar fractions of oxygen $Y$ and the Darcy velocity $u$.

In some works, e.g., [16], the system 1-5 is written in terms of mass densities instead of molar densities. It comes with two problems. First, one has to take into account that molar mass depends on gas mixture composition. The second problem is related to the term $c_{\mathrm{g}} \rho$ in Eq. 1, which is the average heat capacity of the gases in the mixture at constant pressure. As $\mathrm{O}_{2}$ and $\mathrm{CO}_{2}$ are linear molecules, they have the same number of degrees of freedom, and their molar heat capacity (at constant pressure) is practically the same. When the reaction can be regarded as being $\mathrm{C}+\mathrm{O}_{2} \rightarrow \mathrm{CO}_{2}$, the total number of moles in the gas does not change during the reaction. Therefore, as $\mathrm{O}_{2}$ reacts and is replaced by the same number of moles of $\mathrm{CO}_{2}$, the average molar heat capacity of the mixture stays (practically) constant. This is not the case if the specific heat of the mixture is written in terms of mass rather than in terms of moles, see, e.g., [6].

\subsection{Dimensionless equations}

The equations are non-dimensionalized by introducing dimensionless dependent and independent variables (denoted by tildes) as ratios of the dimensional quantities and reference quantities (denoted by stars):

$$
\begin{aligned}
& \tilde{t}=\frac{t}{t^{*}}, \tilde{x}=\frac{x}{x^{*}}, \tilde{\theta}=\Delta \tilde{T}=\frac{T-T_{\mathrm{res}}}{\Delta T^{*}}, \tilde{\rho}=\frac{\rho}{\rho^{*}}, \\
& \tilde{\rho}_{\mathrm{f}}=\frac{\rho_{\mathrm{f}}}{\rho_{\mathrm{f}}^{*}}, \tilde{u}=\frac{u}{u^{*}}, \tilde{Y}=\frac{Y}{Y^{*}} .
\end{aligned}
$$

Our choice for reference quantities is

$$
\begin{aligned}
t^{*} & =\frac{1}{k_{\mathrm{p}} Y_{\text {inj }}} \exp \left(\frac{E_{\mathrm{r}}}{R T_{\text {res }}}\right), v^{*}=\frac{Y_{\text {inj }} \rho^{*} u_{\text {inj }}}{\rho_{\mathrm{f}}^{\text {res }}}, x^{*}=v^{*} t^{*}, \\
\Delta T^{*} & =\frac{Q_{\mathrm{r}} \rho_{\mathrm{f}}^{\text {res }}}{C_{\mathrm{m}}}, \rho^{*}=\frac{P}{R T_{\mathrm{res}}}, \rho_{\mathrm{f}}^{*}=\rho_{\mathrm{f}}^{\text {res }}, u^{*}=u_{\text {inj }}, Y^{*}=Y_{\text {inj }},
\end{aligned}
$$

where $T_{\text {res }}$ and $\rho_{\mathrm{f}}^{\text {res }}$ are the initial reservoir temperature and molar density of fuel, $u_{\text {inj }}$ is the injection gas velocity, and $Y_{\text {inj }}$ is the oxygen molar fraction in the injected gas. In Eq. 8, $v^{*}$ turns out to be the combustion wave speed as it is shown later; $t^{*}$ is the characteristic time for fuel combustion at the initial reservoir temperature $T_{\text {res }} ; \Delta T^{*}$ is the deviation of peak temperature from reservoir temperature, for the case of complete combustion of fuel under adiabatic conditions. Typical values for parameters in Eq. 8 are presented in Section 5 . 
Using Eqs. 7 and 8 and omitting the tildes, Eqs. 1-6 are written in dimensionless form as:

$$
\begin{aligned}
\frac{\partial \theta}{\partial t}+V_{T} \frac{\partial(\rho u \theta)}{\partial x} & =\frac{1}{\operatorname{Pe}_{T}} \frac{\partial^{2} \theta}{\partial x^{2}}+\Phi, \\
\frac{\partial \rho}{\partial t}+\sigma \frac{\partial(\rho u)}{\partial x} & =0, \\
\frac{\partial(Y \rho)}{\partial t}+\sigma \frac{\partial(Y \rho u)}{\partial x} & =\frac{1}{\operatorname{Pe}} \frac{\partial}{\partial x}\left(\rho \frac{\partial Y}{\partial x}\right)-\sigma \Phi, \\
\frac{\partial \rho_{\mathrm{f}}}{\partial t} & =-\Phi, \\
\rho & =\theta_{0} /\left(\theta+\theta_{0}\right),
\end{aligned}
$$

$\Phi=t^{*} W_{\mathrm{r}} / \rho_{\mathrm{f}}^{*}=Y \rho_{\mathrm{f}} \exp \left(\frac{\mathcal{E}}{\theta_{0}}-\frac{\mathcal{E}}{\theta+\theta_{0}}\right)$,

with dimensionless constants

$$
\begin{aligned}
\mathrm{Pe}_{T} & =\frac{v^{*} x^{*} C_{\mathrm{m}}}{\lambda}, \quad V_{T}=\frac{c_{\mathrm{g}} \rho_{\mathrm{f}}^{\text {res }}}{C_{\mathrm{m}} Y_{\mathrm{inj}}}, \quad \sigma=\frac{\rho_{\mathrm{f}}^{\text {res }}}{\varphi Y_{\mathrm{inj}} \rho^{*}}, \\
\mathrm{Pe} & =\frac{v^{*} x^{*}}{D}, \quad \mathcal{E}=\frac{E_{\mathrm{r}}}{R T^{*}}, \quad \theta_{0}=\frac{T_{\mathrm{res}}}{T^{*}} .
\end{aligned}
$$

Here $\mathrm{Pe}_{T}$ and $\mathrm{Pe}$ are the Peclet numbers for thermal and mass diffusion; $\sigma$ is the fuel to oxygen concentration rate; $V_{T}$ turns out to be the dimensionless thermal wave speed as explained later, $\mathcal{E}$ is the scaled activation energy, and $\theta_{0}$ is the scaled reservoir temperature. Typical values of the quantities in Eq. 15 are given in Section 5. The system must be solved with the initial reservoir conditions

$t=0, x \geq 0: \quad \theta=Y=0, \quad \rho_{\mathrm{f}}=1$,

and the injection conditions

$t \geq 0, x=0: \quad \theta=0, \quad Y=u=1, \quad \rho_{\mathrm{f}}=0$.

\section{Thermal and combustion waves}

The solution of system 9-13 with general data for large times essentially takes the form of a sequence of waves, which can be thermal, combustion, gas, or fuel composition waves, see [16]. Particularly with conditions 16 and 17, there are only thermal and combustion waves. In typical in situ applications, the thermal wave is slower than the combustion wave, see, e.g., [14]. We will consider this case from now on.

In the thermal wave, which is upstream of the combustion wave, the fuel concentration and reaction rate are $\rho_{\mathrm{f}}=\Phi=0$ (burned state). Assuming that $\sigma$ is large, which is always the case for gas injection, we can neglect small time-derivative terms in Eqs. 10 and 11, see, e.g., [5]. Then we find that the gas flux and oxygen fraction are constant in the thermal wave and equal to their values at the injection point: $\rho u=1$ and $Y=1$, see Eqs. 17 and 13. The temperature varies in the thermal wave from $\theta=0$ upstream to $\theta^{b}>0$ downstream of the wave. We remark that Eq. 9 with $\rho u=1$ and with $\Phi$ replaced by zero has a solution in the form of a heat wave, which expands in time due to thermal longitudinal conduction and propagates with speed $V_{T}$, see, e.g., [15].

We are looking for the combustion wave as a traveling wave moving with positive speed $V$. All quantities in the wave depend on the traveling coordinate $\xi=$ $x-V t$. The limiting states of the combustion wave are

$\xi \rightarrow+\infty: \quad \theta^{u}=Y^{u}=0, \quad u^{u}>0, \quad \rho_{\mathrm{f}}^{u}=1$,

$\xi \rightarrow-\infty: \quad \theta^{b}>0, \quad Y^{b}=(\rho u)^{b}=1, \quad \rho_{\mathrm{f}}^{b}=0$,

where the superscript $u$ denotes the unburned (downstream) state and the superscript $b$ denotes the burned (upstream) state. Here the state Eq. 18 corresponds to the initial reservoir state Eq. 16, and the state Eq. 19 differs from the injection condition Eq. 17 because temperature changes in the thermal wave. The unknown quantities in the combustion wave are $\theta^{b}, u^{u}$ and the wave speed $V$.

Traveling wave equations are obtained from Eqs. 912 by replacing $\partial / \partial t$ by $-V d / \mathrm{d} \xi$ and $\partial / \partial x$ by $d / \mathrm{d} \xi$. They become

$$
\begin{aligned}
-V \theta^{\prime}+V_{T}(\rho u \theta)^{\prime} & =\theta^{\prime \prime} / \mathrm{Pe}_{T}+\Phi, \\
-V \rho^{\prime}+\sigma(\rho u)^{\prime} & =0, \\
-V(Y \rho)^{\prime}+\sigma(Y \rho u)^{\prime} & =\left(\rho Y^{\prime}\right)^{\prime} / \mathrm{Pe}-\sigma \Phi, \\
-V \rho_{\mathrm{f}}^{\prime} & =-\Phi,
\end{aligned}
$$

where the prime denotes the derivative with respect to $\xi$. As it was mentioned before, $\sigma$ is typically large for gas injection, and it is usual to neglect the small terms $-V \rho^{\prime}$ and $-V(Y \rho)^{\prime}$ in Eqs. 21 and 22 (the calculations taking these terms into account were made in [6], yielding essentially the same results). Under these simplifications, we substitute $\Phi$ from Eq. 23 into Eqs. 20 and 22 and rewrite Eqs. 20-23 as

$$
\begin{aligned}
\left(\theta^{\prime} / \mathrm{Pe}_{T}+\left(V-V_{T} \rho u\right) \theta+V \rho_{\mathrm{f}}\right)^{\prime} & =0, \\
(\sigma \rho u)^{\prime} & =0, \\
\left(\rho Y^{\prime} / \mathrm{Pe}-\sigma Y \rho u-\sigma V \rho_{\mathrm{f}}\right)^{\prime} & =0, \\
V \rho_{\mathrm{f}}^{\prime} & =\Phi .
\end{aligned}
$$


Notice that the highest-order derivative terms containing $1 / \mathrm{Pe}_{T}$ and $1 / \mathrm{Pe}$ are small almost everywhere, except around the combustion front, where they may be large because thermal diffusion and mass diffusion play an important role. That is why such terms are not neglected in singular perturbation theory of ordinary differential equations.

Integrating Eqs. 24-26 between $-\infty<\xi<\infty$, using Eqs. 13,18 , and 19, and the conditions $\theta^{\prime}=Y^{\prime}=0$ at the limiting states $\xi \rightarrow \pm \infty$, we obtain

$\theta^{b}=1 /\left(1-V_{T}\right), \quad u^{u}=1, \quad V=1$.

Notice that the condition $\theta^{b}>0$ is equivalent to the requirement $V_{T}<1=V$, i.e., the thermal wave must be slower than the combustion wave. This condition is typically satisfied due to the large heat capacity of porous rock compared to gas heat capacity, see Eq. 15 . Conditions 28 for complete consumption of fuel and oxygen are well-known, see, e.g., [15-17]. These relations are equivalent to the Rankine-Hugoniot conditions for shocks. From now on, our goal is to compute the traveling profile of the combustion wave, i.e., the dependence of all variables on $\xi$.

Integrating Eqs. $24-26$ from $\xi$ to $\infty$ and using Eqs. 18,23 , and 28 yield

$$
\begin{aligned}
\theta^{\prime} / \mathrm{Pe}_{T} & =1-\rho_{\mathrm{f}}-\theta / \theta^{b}, \\
u & =1 / \rho=\left(\theta+\theta_{0}\right) / \theta_{0}, \\
Y^{\prime} /(\sigma \mathrm{Pe}) & =\left(Y+\rho_{\mathrm{f}}-1\right)\left(\theta+\theta_{0}\right) / \theta_{0} .
\end{aligned}
$$

Here Eq. 30 was used in the derivation of Eqs. 29 and 31. Equations 29-31 together with Eq. 27 determine the traveling wave profile. These equations will be studied numerically in Section 5. In the case of small thermal and mass diffusion, an analytical approach for the computation of the wave profile will be developed in the section below.

\section{Asymptotic expansion for the combustion wave for small diffusion coefficients}

Let us rewrite Eqs. 29 and 31 using $V=1$ from Eqs. 28 and 27 using Eq. 14. We obtain

$$
\begin{aligned}
\epsilon \frac{\mathrm{d} \theta}{\mathrm{d} \hat{\xi}} & =\frac{1}{\operatorname{Pe}_{T}} \frac{\mathrm{d} \theta}{\mathrm{d} \xi}=1-\rho_{\mathrm{f}}-\theta / \theta^{b}, \\
\epsilon \frac{\mathrm{d} Y}{\mathrm{~d} \hat{\xi}} & =\frac{1}{\operatorname{Pe}_{T}} \frac{\mathrm{d} Y}{\mathrm{~d} \xi}=\frac{\sigma \mathrm{Pe}}{\operatorname{Pe}_{T} \theta_{0}}\left(Y+\rho_{\mathrm{f}}-1\right)\left(\theta+\theta_{0}\right), \\
\frac{\mathrm{d} \rho_{\mathrm{f}}}{\mathrm{d} \hat{\xi}} & =\frac{1}{\epsilon \mathrm{Pe}_{T}} \frac{\mathrm{d} \rho_{\mathrm{f}}}{\mathrm{d} \xi}=Y \rho_{\mathrm{f}} \exp \left(\frac{\mathcal{E}}{\theta^{b}+\theta_{0}}-\frac{\mathcal{E}}{\theta+\theta_{0}}\right),
\end{aligned}
$$

where we introduced the scaled coordinate $\hat{\xi}=\mathrm{Pe}_{T} \epsilon \xi$ with

$\epsilon=\frac{1}{\mathrm{Pe}_{T}} \exp \left(\frac{\mathcal{E}}{\theta_{0}}-\frac{\mathcal{E}}{\theta^{b}+\theta_{0}}\right)$.

The right-hand sides of Eqs. 32-34 do not contain small parameters. In particular, $\sigma \mathrm{Pe} / \mathrm{Pe}_{T}$ is usually large due to the large values of $\sigma$. Therefore, we can look for a solution as an asymptotic expansion, assuming that $\epsilon \ll$ 1 . This hypothesis is valid in some practically important cases as will be shown in Section 5, where $\epsilon \approx 0.0041$ and $\sigma \mathrm{Pe} / \mathrm{Pe}_{T} \approx 38.93$.

The zero-order approximation in the asymptotic expansion is found by setting $\epsilon=0$ in Eqs. 32 and 33, which yields

$\theta=\left(1-\rho_{\mathrm{f}}\right) \theta^{b}, \quad Y=1-\rho_{\mathrm{f}}$.

Substituting Eq. 36 into Eq. 34, we obtain

$$
\frac{\mathrm{d} \rho_{\mathrm{f}}}{\mathrm{d} \hat{\xi}}=\left(1-\rho_{\mathrm{f}}\right) \rho_{\mathrm{f}} \exp \left(\frac{\mathcal{E}}{\theta^{b}+\theta_{0}}-\frac{\mathcal{E}}{\left(1-\rho_{\mathrm{f}}\right) \theta^{b}+\theta_{0}}\right) .
$$

The Eq. 37 can be solved for $\hat{\xi}$ in terms of $\rho_{\mathrm{f}}$ using the exponential integral function $\mathrm{E}_{1}(x)=\int_{x}^{\infty} t^{-1} e^{-t} \mathrm{~d} t$ (see [1]) as

$\hat{\xi}=\hat{\xi}_{0}+\mathrm{E}_{1}(a-\eta) \exp (a-b)-\mathrm{E}_{1}(b-\eta)$,

where

$a=\frac{\mathcal{E}}{\theta_{0}}, \quad b=\frac{\mathcal{E}}{\theta^{b}+\theta_{0}}, \quad \eta=\frac{\mathcal{E}}{\left(1-\rho_{\mathrm{f}}\right) \theta^{b}+\theta_{0}}$,

and $\hat{\xi}_{0}$ is an arbitrary constant reflecting translational symmetry of the traveling wave solution. The solution Eqs. 38 and 39 can be verified by substituting into Eq. 37. Notice that $b<\eta<a$, so that $b-\eta<0$ in the second exponential integral function in Eq. 38. For negative $x$, we omit imaginary part $-i \pi$ in the function $\mathrm{E}_{1}(x)$, as it can always be included in $\hat{\xi}_{0}$, see [1].

In the original coordinate $\xi=\hat{\xi} /\left(\mathrm{Pe}_{T} \epsilon\right)$, expressions 38,39 , and 35 yield

$\xi=\xi_{0}+\mathrm{E}_{1}(a-\eta)-\mathrm{E}_{1}(b-\eta) \exp (b-a)$.

The solution of systems 32-34 can be represented as an asymptotic series $\theta=\theta^{0}+\epsilon \theta^{1}+\cdots, Y=Y^{0}+$ $\epsilon Y^{1}+\cdots$, and $\rho_{\mathrm{f}}=\rho_{\mathrm{f}}^{0}+\epsilon \rho_{\mathrm{f}}^{1}+\cdots$, where $\theta^{0}(\hat{\xi}), Y^{0}(\hat{\xi})$, and $\rho^{0}(\hat{\xi})$ are zero-order approximations given by Eqs. 36, 38, and 39. In the Appendix, explicit formulae for the correction terms $\theta^{1}, Y^{1}, \rho_{\mathrm{f}}^{1}$ are derived. 


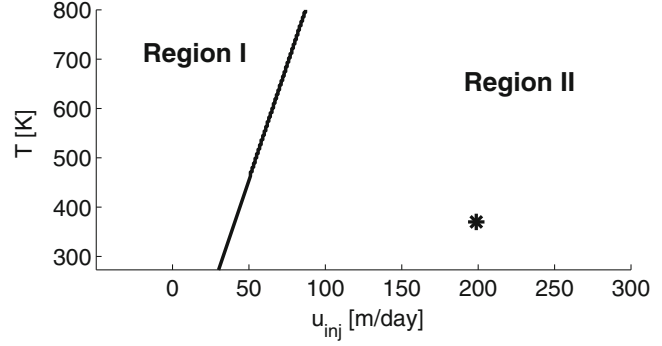

Fig. 2 The region II corresponds to points in the parameter space $\left(u_{\text {inj }}, T_{\text {res }}\right)$ where $\epsilon<0.1$ (other parameters are taken from Table 1). It shows approximately the region, where the asymptotic expansion of Section 4 and of the Appendix is applicable. The solid point corresponds to $u_{\text {inj }}$ and $T_{\text {res }}$ in Table 1

\section{Numerical example}

In this section, we present numerical results by using the values of parameters in Table 1 . These values correspond to low-temperature oxidation reaction of the oil initially present at low concentration in the reservoir. We count moles of fuel as if each molecule has only one carbon atom. The rate of low-temperature oxidation varies a lot among different oils. The reaction rate parameters given in Table 1 are compatible with the experimental data for oil in [11].

As we have shown in Section 4, in order to apply the singular perturbation method to system 29, 31, and 27, the parameter $\epsilon$ defined in Eq. 35 must be small. We verify this condition for practical ranges of the reservoir temperature $T_{\text {res }}$ and of the injected gas speed $u_{\text {inj; }}$; the results are shown in Fig. 2. On the plot, the region II corresponds to the case when $\epsilon<0.1$.

Now the results described in previous sections are verified numerically. The parameter values from Table 1 are used. Expressions 8 and 15 yield the following values of reference and dimensionless parameters

$$
\begin{aligned}
x^{*} & =62.9(\mathrm{~m}), \quad t^{*}=1.463 \times 10^{6}(\mathrm{~s}), \quad \Delta T^{*}=74.4(\mathrm{~K}), \\
v^{*} & =4.3 \times 10^{-5}(\mathrm{~m} / \mathrm{s}) \quad \text { or } 3.695(\mathrm{~m} / \text { day }), \\
\mathrm{Pe}_{T} & =6180, V_{T}=0.0243, \sigma=179, \mathrm{Pe}=1344 \\
\mathcal{E} & =93.8, \theta_{0}=4.97 .
\end{aligned}
$$

The zero-order approximation is obtained using Eq. 40. Then the first-order approximations can be computed as described in the Appendix. The results are presented in Fig. 3. Notice that the first-order correction is very small, around $1 \%$, so the approximation is expected to be very accurate. The characteristic length $x^{*}=62.9 \mathrm{~m}$ of the combustion wave is large, since it is determined by the slow reaction at the reservoir temperature, see Eqs. in 8.

In order to solve numerically the original systems 913, we use a splitting method with adaptative time step. For this purpose, we combine Eq. 10 with Eq. 9 and use $\rho$ given by Eq. 13 yielding

$$
\begin{aligned}
\frac{1}{\mathrm{Pe}_{T}} \frac{\partial^{2} \theta}{\partial x^{2}} & -V_{T} \frac{\partial}{\partial x}\left(\frac{\theta_{0} u \theta}{\theta+\theta_{0}}\right)-\frac{\sigma}{\theta_{0}}\left(\theta+\theta_{0}\right)^{2} \frac{\partial}{\partial x}\left(\frac{\theta_{0} u}{\theta+\theta_{0}}\right) \\
+ & \Phi=0 .
\end{aligned}
$$

\section{Summary of the splitting method:}

1. Initialize the variables $\theta, Y$, and $\rho_{\mathrm{f}}$ for $t=0$ as given in Eq. 16.

2. Initialize $u$ by direct integration of Eq. 43 .

3. Apply the classical Crank-Nicolson scheme to Eqs. 9, 11, and 12, in order to obtain $\theta, Y$, and $\rho_{\mathrm{f}}$ at the new time. Here $\rho$ is given by Eq. 13. Use

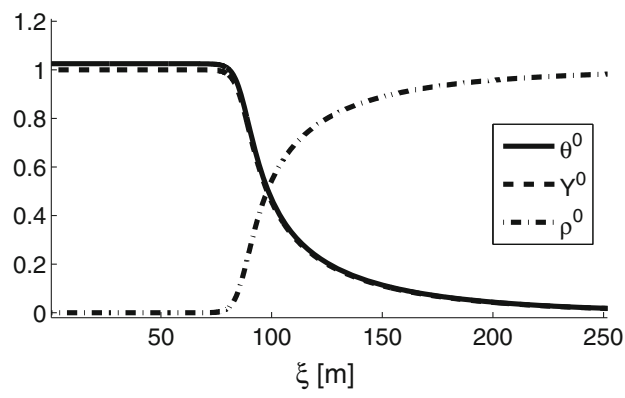

Fig. 3 Zero-order approximation of the combustion traveling wave is shown on the left. The first order correction is shown on the right. As the reservoir temperature and injection speed belong to the region II in Fig. 2, the first-order correction terms

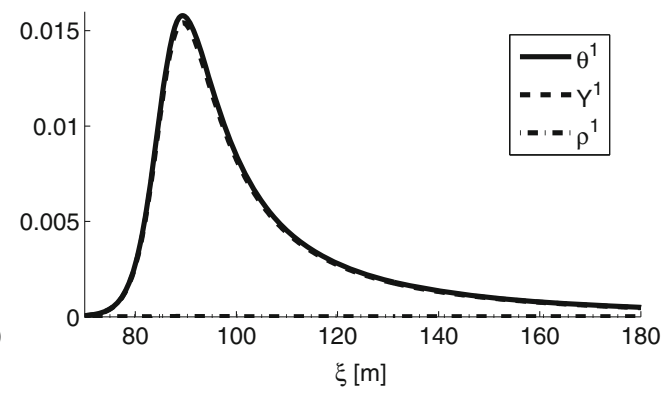

are small. Both figures have horizontal scale in meters; in the vertical scale, we show dimensionless quantities $\theta, Y$, and $\rho_{\mathrm{f}}$ defined in Eq. 7 


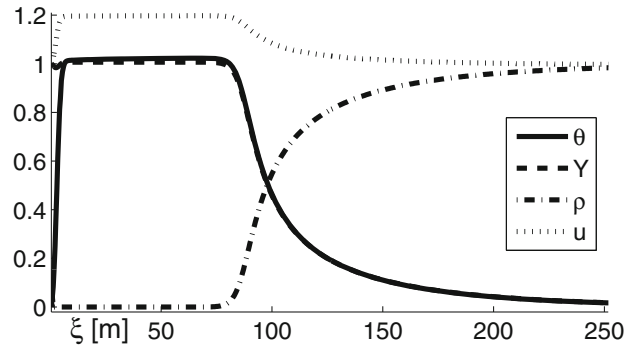

Fig. 4 On the left, we show numerical simulation results for time $t=2.6 \times 10^{6} \mathrm{~s}$. On the right, we compare the combustion wave profile with the zero-order asymptotic approximation of the solution. Circles represent numerical simulation results and

Newton's method to solve the non-linear CrankNicolson step taking into account the left boundary Dirichlet conditions Eq. 17 and the zero Neumann conditions on the right.

4. As in step 2, calculate $u$ by direct integration of Eq. 43.

5. Adapt the size of the future time step using information about convergence of the Newton's method.

- Repeat steps 3, 4, and 5 until the final time is reached.

The simulation results were also confirmed using a hybrid method which combines Crank-Nicolson scheme to solve Eqs. 9, 11, and 12 and Box scheme to solve Eq. 10, see [6, 7]. On the left side of Fig. 4, we plot the simulation results at time $t=2.6 \times 10^{6} \mathrm{~s}$. On the right side of the Fig. 4, we compare the simulation results with the combustion wave profile in Fig. 3 given by the analytical formulae for the zero-order approximation.

\section{Conclusion}

We considered the combustion process when air is injected into a porous medium containing immobile fuel and inert gas. Low-temperature oxidation reaction was considered and heat losses were neglected. We assumed that reaction occurs for all temperatures.

A combustion model including heat and mass balance equations for longitudinal flow was considered. We developed a method for computing the traveling wave profile in the form of an asymptotic expansion and derived its zero-order approximation. This wave profiles appears to be different from wave profiles analyzed in other papers, where only the reaction at highest temperatures was taken into account.

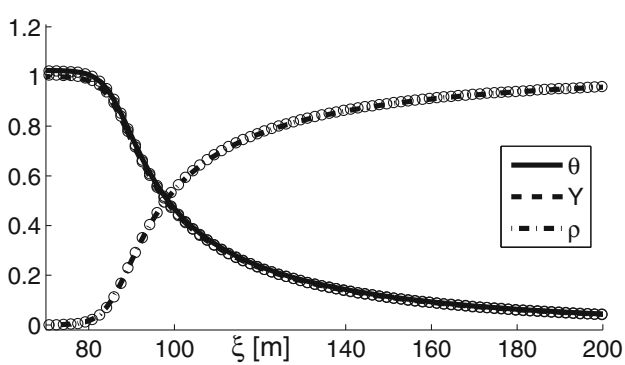

curves the asymptotic solution. Both figures have horizontal scale in meters; in the vertical scale, we show dimensionless quantities $\theta, Y$, and $\rho_{\mathrm{f}}$ defined in Eq. 7

One can see from Figs. 3 and 4 that the traveling wave solution contains a long tail in the downstream zone. The reaction rate is essential for all temperatures, as it can be seen by inspecting the oxygen fraction profile. The existence of such a long tail is an essential feature of our solution, where the heat conduction is assumed to be small and heat losses are negligible. In some cases such as the low-temperature oxidation example considered above, this tail may be reasonably short and the solution is realistic.

When the tail is very long in practice, total heat losses increase, and the heat released by the reaction at low temperatures can be neglected. In such a case, analysis, see, e.g., [2, 3, 14, 15, 17], leads to other parameter values for the combustion wave. In particular, oxygen breakthrough phenomenon is possible. We conclude that, in order to identify the appropriate solution method for each particular case, we should take heat losses into account.

Acknowledgements D.M. is indebted to Yucel Akkutlu for discussions and explanations on the physical model of in situ combustion; he also thanks Stephen Schecter for useful discussions. The authors are grateful to the referee for suggestions on improving the paper. This work was supported in part by CNPq under grants: 301564/2009-4, 472923/2010-2, 490707/20084, 314583/2009-2, and 477907/2011-3; CNPq/INCTmat; and FAPERJ under grants: E-26/110.310/2007, E-26/110.972/2008, E26/102.723/2008, E-26/112.220/2008, and E-26/110.337/2010.

\section{Appendix: The singular perturbation method}

Many different works describe and use singular perturbation methods in order to study multiple scale and stiff problems $[12,13,18]$. Geometric aspects of singularly perturbed differential equation were studied in [10,13]. More details on the application of this method to our problem are given in $[6,8]$. Physical aspects of the resulting approximation are discussed in [9]. 
Here we apply singular perturbation theory in order to determine a heteroclinic orbit for an autonomous system of ordinary differential Eqs. 32-34, which can be written in the general form

$$
\left\{\begin{array}{c}
\epsilon \dot{\mathbf{x}}=\mathbf{f}(\mathbf{x}, y), \\
\dot{y}=g(\mathbf{x}, y)
\end{array}\right.
$$

where $\epsilon$ is a small parameter and the dot denotes the derivative with respect to a real coordinate $t$. The functions $\mathbf{f}=\left(f^{1}, \ldots, f^{n}\right)^{T}$ and $g$ are smooth. The phase space of the system consists of the fast vector $\mathbf{x} \in \mathbb{R}^{n}$ and slow variable $y \in \mathbb{R}$. We assume that Eq. 44 has two isolated equilibria named $(-)$ and $(+)$ :

$$
\begin{gathered}
\mathbf{f}\left(\mathbf{x}^{-}, y^{-}\right)=\mathbf{f}\left(\mathbf{x}^{+}, y^{+}\right)=0 \quad \text { and } \\
g\left(\mathbf{x}^{-}, y^{-}\right)=g\left(\mathbf{x}^{+}, y^{+}\right)=0 .
\end{gathered}
$$

We are interested in finding a heteroclinic orbit $\mathbf{x}(t), y(t)$ of system 44 , which connects $\left(\mathbf{x}^{-}, y^{-}\right)$at $t \rightarrow$ $-\infty$ to $\left(\mathbf{x}^{+}, y^{+}\right)$at $t \rightarrow+\infty$. We consider the case $y^{-} \neq$ $y^{+}$when the slow component $y(t)$ undergoes a finite change.

\subsection{Zero-order approximation}

Let us denote by $\mathbf{x}^{0}(t), y^{0}(t)$ the limit $\epsilon \rightarrow 0$ of the heteroclinic orbit $\mathbf{x}(t), y(t)$. By taking $\epsilon=0$ in system Eq. 44, we obtain

$\mathbf{f}\left(\mathbf{x}^{0}, y^{0}\right)=0$

$\dot{y}^{0}=g\left(\mathbf{x}^{0}, y^{0}\right)$.

Notice that Eq. 46 is a system of $n$ scalar algebraic equations with $n+1$ unknowns. We assume that this system defines a curve $\Gamma_{0}$ in the space $(\mathbf{x}, y)$, which connects the equilibria ( $(-)$ and (+), see Fig. 5a. We also assume that

$\operatorname{det} \mathbf{f}_{\mathbf{x}} \neq 0$ on $\Gamma_{0}$,

where $\mathbf{f}_{\mathbf{x}}=[\partial \mathbf{f} / \partial \mathbf{x}]$ is the Jacobian matrix of the function $\mathbf{f}(\mathbf{x}, y)$ for fixed $y$. By the implicit function theorem, Eq. 46 has a solution $\mathbf{x}^{0}=\varphi\left(y^{0}\right)$ such that the curve $\Gamma_{0}$
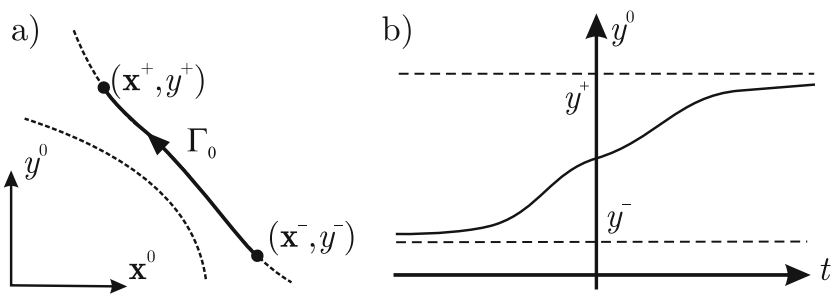

Fig. 5 Heteroclinic orbit in zero-order approximation: a dependence of $\mathbf{x}^{0}$ on $y^{0}$ and $\mathbf{b}$ dependence of $y^{0}$ on $t$ is parametrized as $\left(\varphi\left(y^{0}\right), y^{0}\right)$ for $y^{0}$ between $y^{-}$and $y^{+}$. Of course, $\varphi\left(y^{ \pm}\right)=\mathbf{x}^{ \pm}$.

By substituting $\varphi\left(y^{0}\right)$ into Eq. 47 , we obtain a scalar ODE for $y^{0}$ :

$\dot{y}^{0}=g\left(\mathbf{x}^{0}\left(y^{0}\right), y^{0}\right)$.

According to Eq. $45, g\left(\varphi\left(y^{0}\right), y^{0}\right)=0$ at $y^{0}=y^{ \pm}$. We assume

$g\left(\mathbf{x}^{0}\left(y^{0}\right), y^{0}\right)>0$ for all $y^{-}<y<y^{+}$.

It follows that there exists a solution $y^{0}(t)$ such that $y^{0} \rightarrow y^{ \pm}$as $t \rightarrow \pm \infty$. This solution is a monotone function of $t$, see Fig. 5b. Equation (49) can be solved forward and backward in $t$ with the initial condition $y^{0}(0)$ chosen arbitrarily between $y^{-}$and $y^{+}$(ambiguity is due to the translational invariance of the solution), which proves:

Proposition 1 Let us assume that the equation $\mathbf{f}\left(\mathbf{x}^{0}, y^{0}\right)=0$ determines a curve $\Gamma_{0}=\left(\varphi\left(y^{0}\right), y^{0}\right)$ connecting the equilibria $\left(\mathbf{x}^{-}, y^{-}\right)$to $\left(\mathbf{x}^{+}, y^{+}\right)$, satisfying the hypotheses 48 and 50. Then the asymptotic heteroclinic solution of system 44 in the limit $\epsilon \rightarrow 0$ is given by $\left(\mathbf{x}^{0}(t)=\varphi\left(y^{0}(t)\right), y^{0}(t)\right)$, where $y^{0}(t)$ is a solution of Eq. 49 with the initial condition $y^{0}(0)$ chosen arbitrarily between $y^{-}$and $y^{+}$.

\subsection{Asymptotic series expansion}

The approximate solution given by Proposition 1 is sufficient for many purposes. For example, if $\epsilon$ is very small, then the higher-order approximations contain little useful information. However, in order to increase the accuracy or estimate the error of approximation, one needs higher-order correction terms. Let us look for a solution of system 44 as an asymptotic power series in $\epsilon$ as

$\mathbf{x}(t)=\mathbf{x}^{0}(t)+\epsilon \mathbf{x}^{1}(t)+\epsilon^{2} \mathbf{x}^{2}(t)+\cdots$,
$y(t)=y^{0}(t)+\epsilon y^{1}(t)+\epsilon^{2} y^{2}(t)+\cdots$.

By substituting expansions 51 into the functions $\mathbf{f}(\mathbf{x}, y)$, $g(\mathbf{x}, y)$ and expanding them in a power series in $\epsilon$, we obtain

$\mathbf{f}(\mathbf{x}, y)=\mathbf{f}^{0}+\epsilon \mathbf{f}_{\mathbf{x}} \mathbf{x}^{1}+\epsilon \mathbf{f}_{y} y^{1}+\cdots$,

$g(\mathbf{x}, y)=g^{0}+\epsilon \mathbf{g}_{\mathbf{x}} \mathbf{x}^{1}+\epsilon g_{y} y^{1}+\cdots$. 
Here $\mathbf{f}^{0}=\mathbf{f}\left(\mathbf{x}^{0}, y^{0}\right), g^{0}=g\left(\mathbf{x}^{0}, y^{0}\right)$ and

$\mathbf{f}_{\mathbf{x}}=\left[\begin{array}{ccc}\frac{\partial f^{1}}{\partial x_{1}} & \cdots & \frac{\partial f^{1}}{\partial x_{n}} \\ \vdots & \ddots & \vdots \\ \frac{\partial f^{n}}{\partial x_{1}} & \cdots & \frac{\partial f^{n}}{\partial x_{n}}\end{array}\right], \mathbf{f}_{y}=\left[\begin{array}{c}\frac{\partial f^{1}}{\partial y} \\ \vdots \\ \frac{\partial f^{n}}{\partial y}\end{array}\right]$,

$\mathbf{g}_{\mathbf{x}}=\left[\frac{\partial g}{\partial x_{1}} \cdots \frac{\partial g}{\partial x_{n}}\right], g_{y}=\left[\frac{\partial g}{\partial y}\right]$,

are functions of $t$ evaluated at $\mathbf{x}^{0}(t), y^{0}(t)$. Substituting Eqs. 51 and 52 into Eq. 44, we obtain

$$
\begin{aligned}
\epsilon\left(\dot{\mathbf{x}}^{0}+\epsilon \dot{\mathbf{x}}^{1}+\cdots\right) & =\mathbf{f}^{0}+\epsilon \mathbf{f}_{\mathbf{x}} \mathbf{x}^{1}+\epsilon \mathbf{f}_{y} y^{1}+\cdots \\
\dot{y}^{0}+\epsilon \dot{y}^{1}+\cdots & =g^{0}+\epsilon \mathbf{g}_{\mathbf{x}} \mathbf{x}^{1}+\epsilon g_{y} y^{1}+\cdots .
\end{aligned}
$$

Since, for any $\epsilon, \mathbf{x}(t) \rightarrow x^{ \pm}$and $y(t) \rightarrow y^{ \pm}$as $t \rightarrow \pm \infty$, then the components of expansions 51 must satisfy the following conditions:

$$
\mathbf{x}^{0} \rightarrow x^{ \pm}, y^{0} \rightarrow y^{ \pm}, \mathbf{x}^{k} \rightarrow 0, y^{k} \rightarrow 0 \text { as } t \rightarrow \pm \infty
$$

for all integer $k>0$.

Equating terms with the same power of $\epsilon$, we obtain a series of equations for the unknown functions $\mathbf{x}^{k}(t)$ and $y^{k}(t)$. For the zero-order terms, we recover Eqs. 46 and 47; thus, $\mathbf{x}^{0}(t)$ and $y^{0}(t)$ are given by Proposition 1. Using Eq. 49 for the first-order terms in Eq. 54 yields

$\dot{\mathbf{x}}^{0}=\mathbf{f}_{\mathbf{x}} \mathbf{x}^{1}+\mathbf{f}_{y} y^{1}$,

$\dot{y}^{1}=\mathbf{g}_{\mathbf{x}} \mathbf{x}^{1}+g_{y} y^{1}$.

The solution of this system is given in the following proposition:

Proposition 2 Let us assume that the hypotheses of Proposition 1 are satisfied. Then the first-order terms $\mathbf{x}^{1}(t)$ and $y^{1}(t)$ in the asymptotic expansion 51 have the form

$\mathbf{x}^{1}(t)=\mathbf{f}_{\mathbf{x}}^{-1}\left(\dot{\mathbf{x}}^{0}-\mathbf{f}_{y} y^{1}\right)$,

$y^{1}(t)=-g^{0} \int_{0}^{t} B(\tau) \mathrm{d} \tau, \quad B(t)=\mathbf{g}_{\mathbf{x}} \mathbf{f}^{-2} \mathbf{f}_{y}$.

Proof Let us write Eq. 56 as:

$$
\begin{gathered}
\dot{\mathbf{x}}^{0}-\mathbf{f}_{y} y^{1}=\mathbf{f}_{\mathbf{x}} \mathbf{x}^{1}, \\
\dot{y}^{1}=\mathbf{g}_{\mathbf{x}} \mathbf{x}^{1}+g_{y} y^{1} .
\end{gathered}
$$

Then Eq. 57 follows from Eq. 59. Using Eqs. 46 and 47 and the chain rule, one obtains

$\dot{\mathbf{x}}^{0}=-\mathbf{f}_{\mathbf{x}}^{-1} \mathbf{f}_{y} \dot{y}^{0}=-\mathbf{f}_{\mathbf{x}}{ }^{-1} \mathbf{f}_{y} g^{0}$.
Substituting this expression into Eq. 57 and using the result in Eq. 60, one gets

$\dot{y}^{1}=-\mathbf{g}_{\mathbf{x}} \mathbf{f}_{\mathbf{x}}^{-1}\left(\mathbf{f}_{\mathbf{x}}^{-1} \mathbf{f}_{y} g^{0}+\mathbf{f}_{y} y^{1}\right)+g_{y} y^{1}$.

Now using Eq. 61 with the chain rule, we find the expression

$\dot{g}^{0}=\mathbf{g}_{\mathbf{x}} \dot{\mathbf{x}}^{0}+g_{y} \dot{y}^{0}=-\mathbf{g}_{\mathbf{x}} \mathbf{f}_{\mathbf{x}}{ }^{-1} \mathbf{f}_{y} g^{0}+g_{y} g^{0}$,

which can be used in Eq. 62 to get

$\frac{d}{\mathrm{~d} t}\left(\frac{y^{1}}{g^{0}}\right)=-B$

where $B$ is defined in Eq. 58. Solving Eq. 64, one obtains

$y^{1}=c g^{0}(t)+g^{0}(t) \int_{0}^{t} B(\tau) \mathrm{d} \tau$,

where $c$ is an arbitrary constant. The therm $c g^{0}$ is responsible for infinitesimal time translations of the heteroclinic orbit. Thus, we can take $c=0$, yielding Eq. 58.

Now we have to prove that $\mathbf{x}^{1}$ and $y^{1}$ from Eqs. 57 and 58 satisfy conditions 55 . As $\mathbf{f}_{\mathbf{x}}$ is nonsingular, $B=$ $\mathbf{g}_{\mathbf{x}} \mathbf{f}_{\mathbf{x}}^{-2} \mathbf{f}_{y}$ is a smooth function of $t$ with finite limits as $t \rightarrow \pm \infty$. Thus, $B$ is bounded: $|B(t)|<B_{\max }$ for some $B_{\max }$. Then $y^{1}$ satisfies the inequalities

$$
\begin{aligned}
\left|y^{1}(t)\right| & =\left|g^{0}(t) \int_{0}^{t} B(\tau) \mathrm{d} \tau\right| \leq B_{\max }\left|g^{0}(t) t\right| \\
& \leq B_{\max }\left(\left|g^{0}(t) T\right|+\left|g^{0}(t)(t-T)\right|\right) \leq \\
& \leq B_{\max }\left(\left|g^{0}(t) T\right|+\left|\int_{T}^{t} g^{0}(\tau) \mathrm{d} \tau\right|\right) \\
& \leq B_{\max }\left(\left|g^{0}(t) T\right|+\left|y^{0}(t)-y^{0}(T)\right|\right),
\end{aligned}
$$

where $t$ and $T$ are arbitrary (large) numbers belonging to the interval where $g^{0}(t)$ is monotone by the hypothesis 50 . Now keeping $T$ fixed and taking the limit $t \rightarrow \pm \infty$ in Eq. 66, we obtain $\lim _{t \rightarrow \pm \infty}\left|y^{1}(t)\right| \leq$ $B_{\max }\left|y^{ \pm}-y^{0}(T)\right|$. Since $y^{0}(T) \rightarrow y^{ \pm}$as $T \rightarrow \pm \infty$, then $y^{1} \rightarrow 0$ as $t \rightarrow \pm \infty$. By using this property in Eq. 57 , it is straightforward to show that $\mathbf{x}^{1} \rightarrow 0$ as $t \rightarrow \pm \infty$.

\section{References}

1. Abramowitz, M., Stegun, I.: Handbook of Mathematical Functions with Formulas, Graphs, and Mathematical Tables. Dover, New York (1964)

2. Akkutlu, I., Yortsos, Y.: The dynamics of in-situ combustion fronts in porous media. Combust. Flame 134, 229-247 (2003)

3. Aldushin, A., Rumanov, I., Matkowsky, B.: Maximal energy accumulation in a superadiabatic filtration combustion wave. Combust. Flame 118, 76-90 (1999) 
4. Aldushin, A., Seplyarsky, B.: Propagation of exothermic reaction in a porous-medium during gas blowing. Sov. Phys. Dokl. 23, 483-485 (1978)

5. Bruining, J., Mailybaev, A., Marchesin, D.: Filtration combustion in wet porous medium. SIAM J. Appl. Math. 70, 1157-1177 (2009)

6. Chapiro, G.: Gas-solid combustion in insulated porous media. Ph.D. thesis, IMPA, http://www.preprint.impa.br (2009)

7. Chapiro, G., Hime, G., Mailybaev, A., Marchesin, D., de Souza, A.: Global asymptotic effects of the structure of combustion waves in porous media. In: Hyperbolic Problems: Theory, Numerics, and Applications: Plenary and Invited Talks: Twelfth International Conference on Hyperbolic Problems, 9-13 June 2008, vol. 67, pp. 487-496. AMS (2009)

8. Chapiro, G., Mailybaev, A.A., Marchesin, D., Souza, A.: Singular perturbation in combustion waves for gaseous flow in porous media. In: Proceedings of XXVI CILAMCE, 19-21 October. Guarapari, Brazil (2005)

9. Chapiro, G., Marchesin, D.: Non-diffusive combustion waves in insulated porous media. BJPG 2(2), 18-28 (2008)

10. Fénichel, N.: Geometric singular perturbation theory for ordinary differential equations. J. Differ. Equ. 31, 53-98 (1979)

11. Freitag, N., Verkoczy, B.: Low-temperature oxidation of oils in terms of SARA fractions: why simple reaction models don't work. In: Canadian International Petroleum Conference, Calgary, Alberta (2003)

12. Johnson R., S.: Singular Perturbation Theory, Mathematical Analytical Techniques with Applications to Engineering. Springer, New York (2005)

13. Jones, C.: Geometric singular perturbation theory in dynamical systems. Lect. Notes Math. 1609, 44-118 (1995)

14. Mailybaev, A.A., Bruining, J., Marchesin, D.: Analysis of in situ combustion of oil with pyrolysis and vaporization. Combust. Flame 158, 1097-1108 (2011)

15. Schult, D., Matkowsky, B., Volpert, V., Fernandez-Pello, A.: Forced forward smolder combustion. Combust. Flame 104(12), 1-26 (1996)

16. Souza, A.J., Marchesin, D., Akkutlu, I.Y.: Wave sequences for solid fuel adiabatic in-situ combustion in porous media. Comput. Appl. Math 25(1), 27-54 (2006)

17. Wahle, C., Matkowsky, B., Aldushin, A.: Effects of gas-solid nonequilibrium in filtration combustion. Combust. Sci. Technol. 175, 1389-1499 (2003)

18. Wasow, W.: Asymptotic Expansions for Ordinary Differential Equations. Dover, New York (2002)

19. Zeldovich, Y.B., Barenblatt, G.I., Librovich, V.B., Makhviladze, G.M.: The Mathematical Theory of Combustion and Explosion. Consultants Bureau, New York (1985) 